\title{
Changes in sperm quality and testicular structure in a rat model of type 1 diabetes
}

\author{
Sureeporn Nak-ung', Nutnicha Nakprom', Chonikan Maneengam', Sutisa Nudmamud-Thanoi ${ }^{12}$, \\ Samur Thanoi ${ }^{1,2 * *}$
}

Abstract

Background: Chronic hyperglycemia is a characteristic of diabetes mellitus (DM). Long-lasting hyperglycemia can generate oxidative stress and reactive oxygen species. The effect of this condition on sperm quality and spermatogenesis leads to male infertility and reproductive dysfunction.

Objectives: To investigate changes in sperm quality, morphology of testicular structure, and stage of development of seminiferous tubules in a streptozotocin (STZ)-induced rat model of type 1 DM.

Methods: We divided 15 male Sprague Dawley rats into 2 groups. DM was induced in 7 rats using STZ $(60 \mathrm{mg} / \mathrm{kg}$ intraperitoneally), while the other 8 were treated with citrate buffer as a vehicle control group. Rat semen was collected for quality measurements including motility, normal morphology, and concentration. Morphological changes in testicular structure and stage of development of seminiferous tubules were investigated by histology with hematoxylin and eosin (HE) staining.

Results: Significant decreases in all parameters of sperm quality and testicular weight were found in rats with induced DM. Moreover, abnormal morphology of seminiferous tubules including separation of the germinal epithelium, vacuolization, luminal sloughing of germ cells, and tubular atrophy was increased significantly in these rats, while the proportion of their seminiferous tubules at an early stage of development was significantly higher, but was dramatically decreased in the late stage of development when compared with that in vehicle-treated control rats.

Conclusions: DM has adverse effects on sperm quality, testicular structure, and development of seminiferous tubules. These findings may reflect the male infertility and reproductive dysfunction seen in patients with type 1 DM.

Keywords: diabetes mellitus type 1; infertility; male; reactive oxygen species; spermatogenesis; spermatozoa; sperm maturation; teratozoospermia; testis

Diabetes mellitus (DM) is a metabolic disease resulting from impaired metabolism of carbohydrates, lipids, and proteins. DM can be categorized into 2 major types; type 1 DM (insulin dependent) and type 2 DM (not insulin dependent). The main causes of this disease are a lack of either insulin production or response to insulin, or both, leading to chronic hyperglycemia [1]. Long-lasting hyperglycemia can cause diabetic complications such as vascular disease, diabetic retinopathy, diabetic nephropathy, diabetic neuropathy, and sexual dysfunction [2, 3]. Hyperglycemia can generate oxidative stress and reactive oxygen species (ROS) by several pathways $[2,4]$. Oxidative stress and ROS from these

*Correspondence to: Samur Thanoi, Department of Anatomy, Faculty of Medical Sciences, Naresuan University, Phitsanulok 65000, Thailand,

e-mail: samurt@nu.ac.th

'Department of Anatomy, Faculty of Medical Sciences, Naresuan University, Phitsanulok 65000, Thailand

${ }^{2}$ Centre of Excellence in Medical Biotechnology, Faculty of Medical Sciences, Naresuan University, Phitsanulok 65000, Thailand

¿ Open Access. (c) 2018 Sureeporn Nak-ung et al., published by Sciendo. (c) BY-NC-ND This work is licensed under the Creative Commons Attribution NonCommercial-NoDerivatives 4.0 License. 
pathways are associated with male infertility. Oxidative stress can induce a decrease in testosterone levels, changes in seminiferous tubule structure, and spermatogenesis failure [5-8]. High ROS concentration in semen has been demonstrated in $30 \%-40 \%$ of male infertility [9].

Moreover, there have been reports on changes in sperm morphology including the acrosome, nucleus, mitochondria, and plasma membrane in patients with type 1 DM [10]. Similarly, in animal models of DM, significant decreases in sperm motility, viability, count, and testosterone levels have been shown [11-13].

Streptozotocin (STZ) is a glucose analog produced by Streptomyces achromogenes that is selective for glucose transporter 2 (GLUT2), which is expressed in pancreatic $\beta$-cells, hepatocytes, and renal tubules. STZ induces DNA alkylation and produces ROS, which can lead to pancreatic $\beta$-cell death and impair insulin production. Therefore, STZ has been used to induce type $1 \mathrm{DM}$ in rat models of DM. The present study was designed to investigate sperm quality and morphological changes in seminiferous tubules and their stage of development in a rat model of type $1 \mathrm{DM}$ induced by STZ $[14,15]$.

\section{Materials and methods}

We studied 15 male Sprague Dawley rats from the age of 8 weeks, weighing 250-320 g, in compliance with The Animals for Scientific Purposes Act, BE 2558 (AD 2015) (Thai Government Gazette, Vol. 132, Part 18 a, 13th March 2015) under license after approval by the Center for Animal Research Naresuan University, Phitsanulok, Thailand (approval No. 5801002 ) and in conformance with the revised Institute of Laboratory Animal Resources, Commission on Life Sciences, National Research Council "Guide for the Care and Use of Laboratory Animals" Washington, D.C.: National Academy Press; 1996. All rats were maintained at a constant temperature of $22 \pm 1{ }^{\circ} \mathrm{C}$ under dark light cycle of $12: 12 \mathrm{~h}$ and allowed access to standard laboratory food and water ad libitum. Rats were divided into 2 groups in an unselected manner to include a vehicle control group treated with $0.1 \mathrm{M}$ citrate buffer, $\mathrm{pH} 5$ (control; $\mathrm{n}=8$ ) and a group with STZ-induced type 1 diabetes (DM; $n=7)$. After fasting for $8 \mathrm{~h}$, rats intended for the DM group were injected intraperitoneally with a single dose of STZ (Sigma) $60 \mathrm{mg} / \mathrm{kg}$ in $0.1 \mathrm{M}$ citrate buffer, $\mathrm{pH} 4.5$, and were subsequently found to have a fasting blood glucose level of $\geq 250 \mathrm{mg} / \mathrm{dL}$ and used for the study as a model of DM. At 7-12 weeks after inducing DM, rats were humanely killed by carbon dioxide gas inhalation.

\section{Sperm quality analysis}

The testis and epididymis were removed immediately after death. Sperm from the cauda epididymis were mixed in prewarmed phosphate-buffered saline (PBS), and sperm motility was determined using a Makler counting chamber $[16,17]$. The sperm sample was fixed in $10 \%$ neutral-buffered formalin, and sperm concentration was determined using hemocytometer under a light microscope (400× magnification). Subsequently, the percentage of normal spermatozoa was calculated from a total of 200 spermatozoa under a light microscope after staining with eosin [17-20].

\section{Testicular weight and testicular structure analysis}

Rats were weighed to determine their body weight. After death, their testes were removed and weighed to calculate the testicular weight as a percentage of body weight. Then, testicular tissue was fixed in 10\% neutral-buffered formalin. The tissue was embedded in paraffin wax and sectioned at a thickness of $5 \mu \mathrm{m}$. All sections were stained with hematoxylin and eosin (HE) and the diameter of seminiferous tubules measured in 4 arbitrary areas of tubules measured using ImageJ software (version 1.46r; U.S. National Institutes of Health). Then, 2 sections per rat were examined for normal morphology and morphological changes of seminiferous tubules including separation of the germinal epithelium, vacuolization, luminal sloughing of germ cells, irregular tube, and atrophy of the tubules. The morphological changes were calculated as percentages of morphological change in seminiferous tubules per total tubules [17]. Then, 2 sections per rat were examined to determine the developmental stage of the seminiferous epithelium [21, 22].

\section{Statistical analyses}

Data were analyzed using SPSS Statistics for Windows (version 17.0) by independent sample $t$ tests and are expressed as mean \pm standard error of the mean (SEM). Differences between data were considered significant when the results of the $t$ tests were at $P<0.05$.

\section{Results}

The sperm motility, normal morphology, and concentration in rats in the DM group were significantly decreased when compared with that of rats in the vehicle control group (Figure 1). 

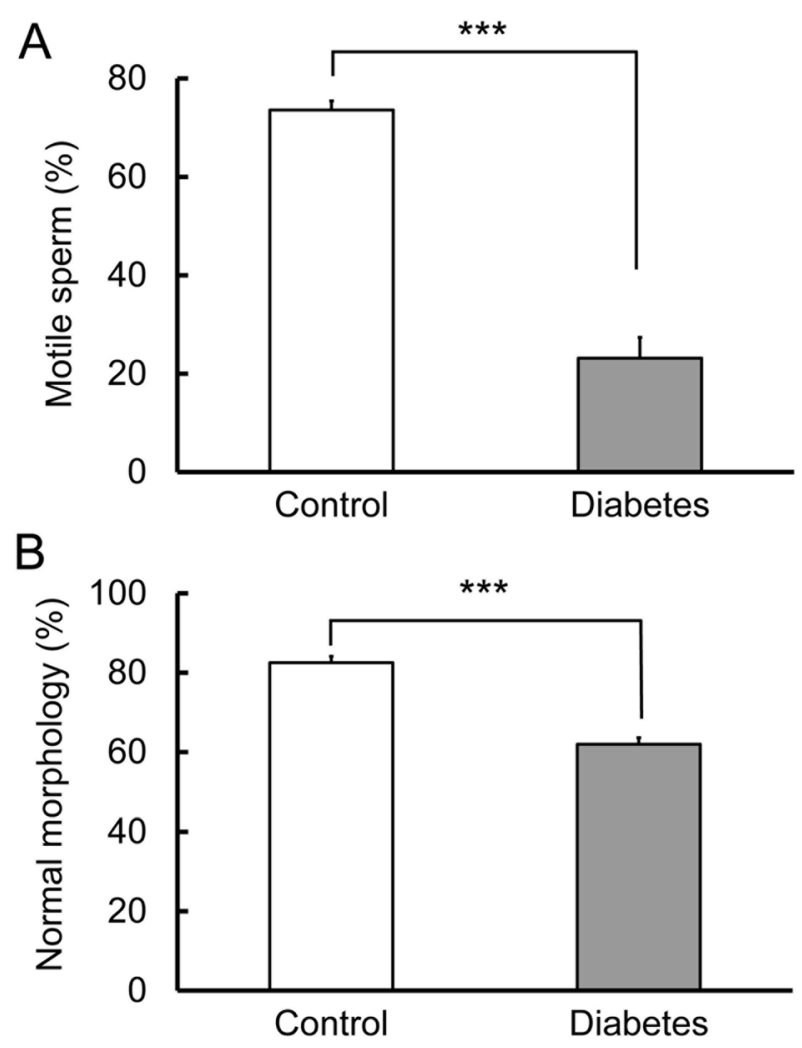

C

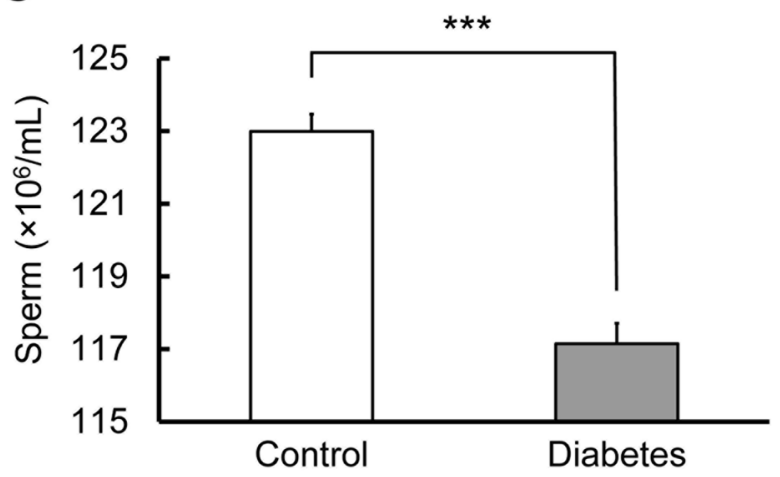

Figure 1. Sperm quality parameters in rats in the group with streptozotocin-induced diabetes mellitus (gray bars) compared with those in vehicle treated rats in the control group (unfilled bars). (A) Percentage of sperm motility. (B) Percentage of normal sperm morphology. (C) Sperm concentration. Bars represent mean percentages or concentrations, and error bars represent standard error of the mean; $t$ test ${ }^{* * *} P<0.001$

A significant reduction in the body weight and testicular weight was found in rats in the DM group, while the testicular weight percentage of body weight was significantly increased in rats in the DM group (Table 1).

The diameter of seminiferous tubules was significantly decreased in rats in the DM group (Figure 2).

We observed morphological changes in seminiferous tubules from the appearance of the tubules in vehicle-treated
Table 1. Body weight, testicular weight, and the testicular weight percentage of body weight in rats in rats in the group with streptozotocin-induced diabetes mellitus and in the vehicle treated control group

\begin{tabular}{lccc}
\hline & $\begin{array}{c}\text { Control group } \\
(\mathbf{n}=\mathbf{8})\end{array}$ & $\begin{array}{c}\text { Diabetic group } \\
(\mathbf{n}=\mathbf{7})\end{array}$ & $\boldsymbol{P}$ \\
\hline Body weight (g) & $453 \pm 5.6$ & $268 \pm 18.8^{* * *}$ & $<0.001$ \\
Testis weight (g) & $2.1 \pm 0.01$ & $1.8 \pm 0.10^{*}$ & 0.02 \\
$\begin{array}{l}\text { Testicular weight as } \\
\text { \%body weight }\end{array}$ & $0.45 \pm 0.01$ & $0.68 \pm 0.05^{* *}$ & 0.003 \\
\hline
\end{tabular}

Values are reported as mean \pm SEM. $t$ test ${ }^{*} P<0.05 ;{ }^{* *} P<0.01 ;{ }^{* * *} P<0.001$ Diabetic, diabetes mellitus; SEM, standard error of the mean

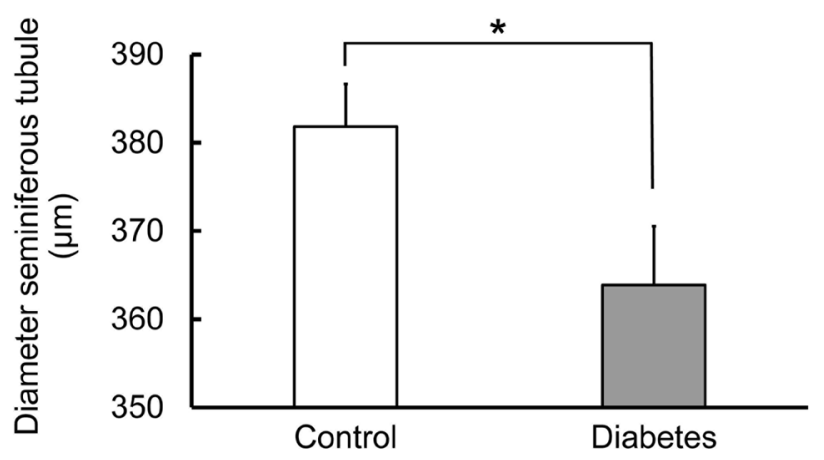

Figure 2. The diameter of seminiferous tubules in rats with streptozotocin-induced diabetes mellitus (gray bars) compared with that in vehicle treated rats in the control group (unfilled bars). Bars represent mean diameters, and error bars represent standard error of the mean; $t$ test $* P<0.05$

control rats (Figure 3A) including separation of the germinal epithelium, vacuolization, luminal sloughing of germ cells, irregular tube, and atrophy in rats in the DM group (Figure 3B-F).

Significant increases in the separation of germinal epithelium, vacuolization, luminal sloughing of germ cells, and atrophy of seminiferous tubules were found in rats in the DM group when the percentages of these morphological abnormalities were compared quantitatively with those in vehicle treated rats in the control group (Figure 4).

The mean proportion of early stage (stage I-V) seminiferous tubules was significantly increased in rats in the DM group when compared with the proportion in rats in the vehicle control group. By contrast, significant decreases in the mean proportion of late stage (stage IX-XIV) tubules were seen in rats in the DM group (Figure 5).

\section{Discussion}

After induction of DM with STZ, the resulting rats with type 1 DM showed abnormal sperm quality including decreases in 

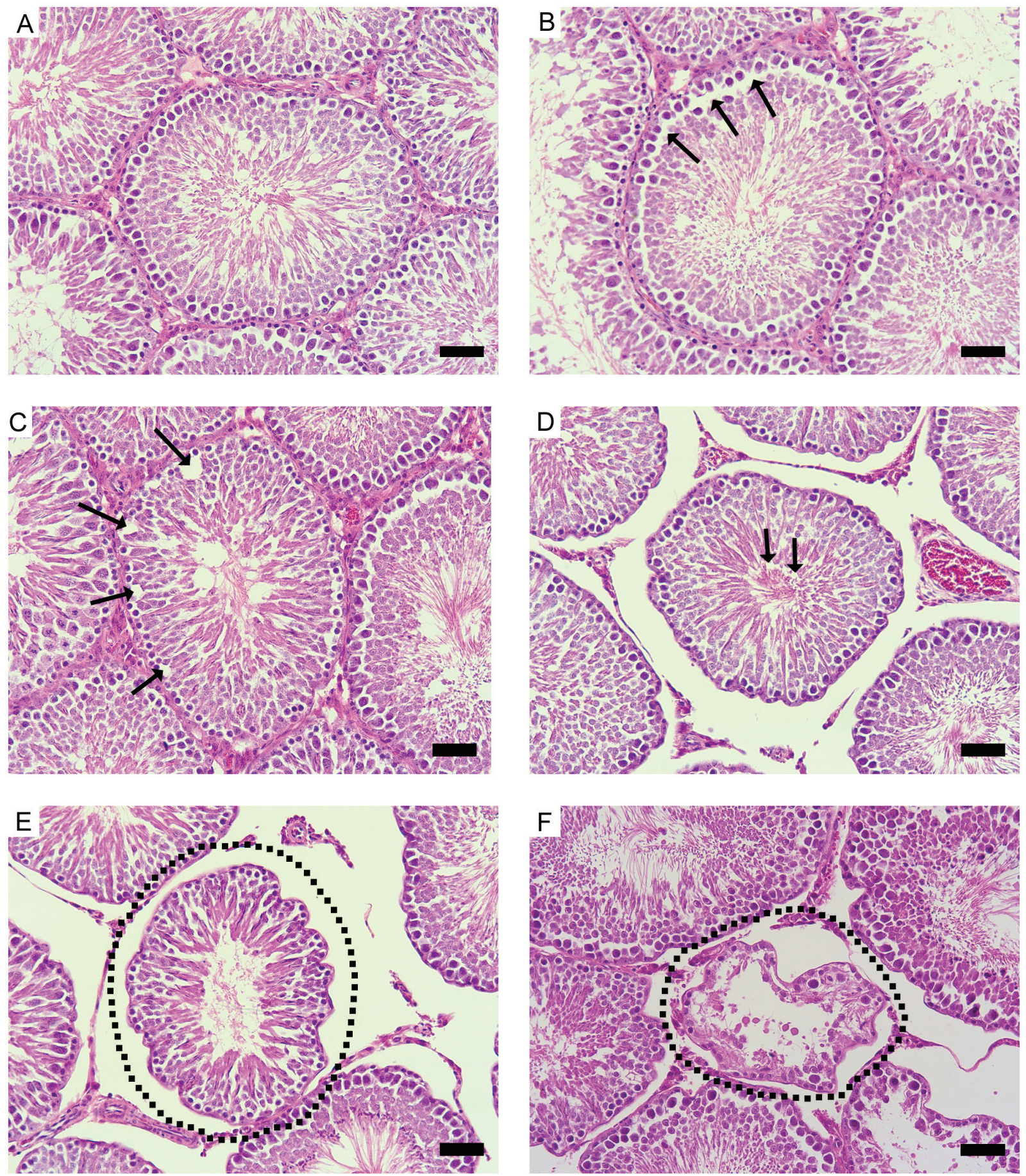

Figure 3. Seminiferous tubule morphology. (A) Normal morphology in vehicle-treated rats. Changes in morphology in rats with streptozotocininduced diabetes mellitus (B) separation of the germinal epithelium (arrows), (C) vacuolization (arrows), (D) luminal sloughing of germ cells (arrows), (E) irregular tube (dashed circle), and (F) atrophy (dashed circle). Scale bars represent $50 \mu \mathrm{m}$ (magnification 200x)

sperm motility, concentration, and normal morphology. These findings are consistent with those in previous studies of sperm quality affected by DM [11, 13, 23]. The decrease in sperm quality may be associated with hyperglycemia resulting in hyperactive oxidative stress. The plasma membrane of sperm containing high levels of polyunsaturated fatty acids and lacking anti-ROS enzymes may result in elimination of nuclear and mitochondrial DNA in sperm as a consequence of lipid peroxidation, which is implicated in sperm defects and apoptosis [24, 25]. 


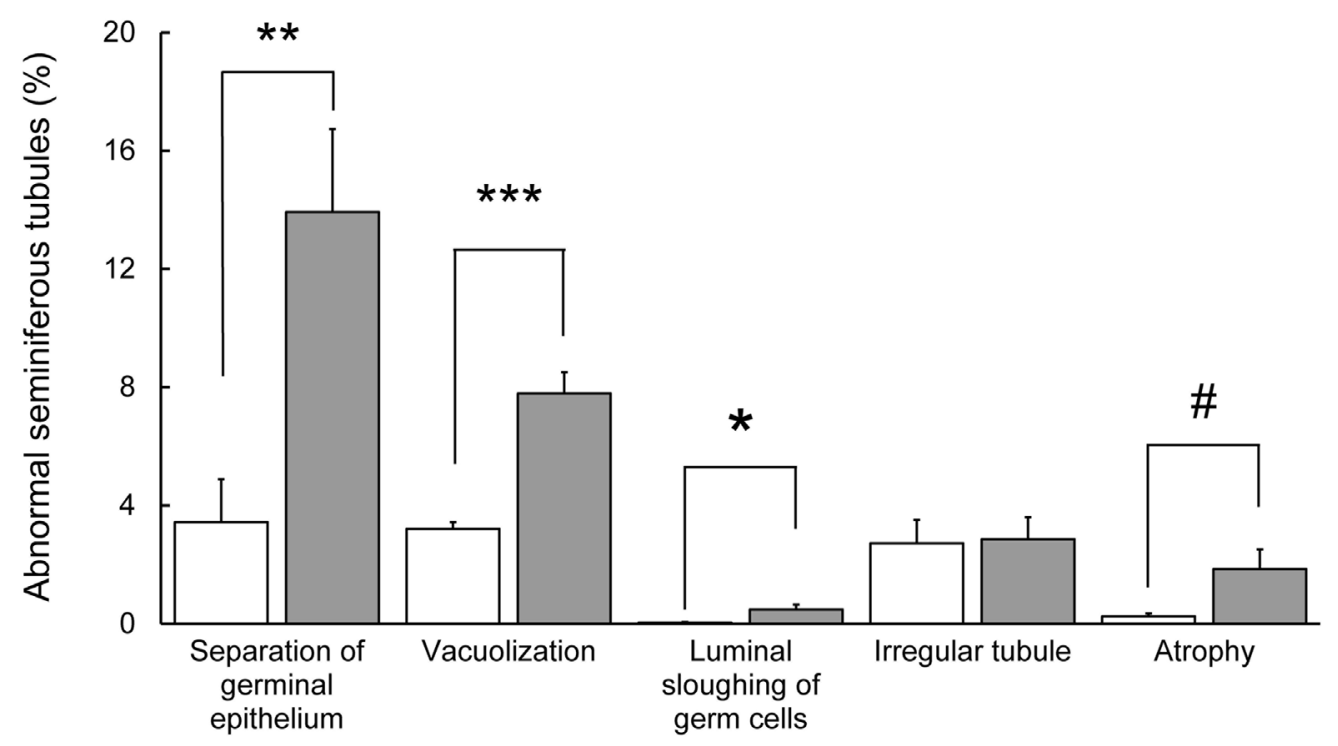

Figure 4. Morphological changes of seminiferous tubules in rats with streptozotocin-induced diabetes mellitus (gray bars) compared with those in vehicle-treated rats in the control group (unfilled bars). Bars represent mean percentages of abnormalities, and error bars represent standard error of the mean. $t$ test ${ }^{\#} P<0.1 ;{ }^{*} P<0.05 ;{ }^{* *} P<0.01 ; * * * 0.001$

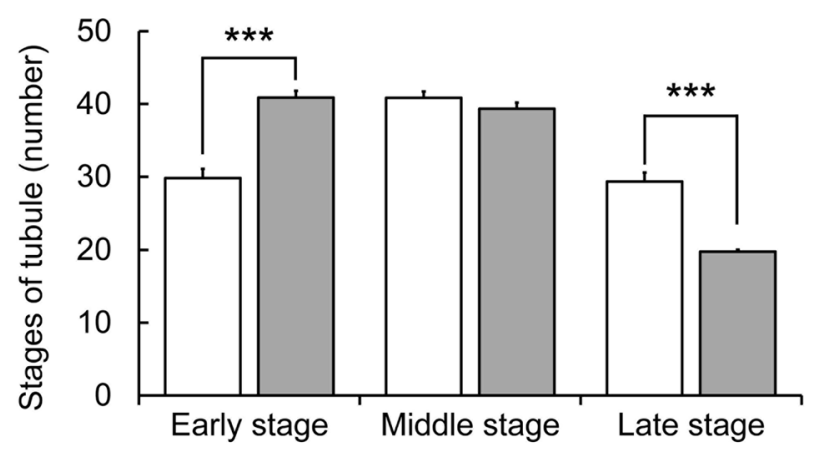

Figure 5. Proportion of seminiferous tubules at various stages of development in rats with streptozotocin-induced diabetes mellitus (gray bars) compared with that in vehicle-treated rats in the control group (unfilled bars). Bars represent mean percentages at the various stages, and error bars represent standard error of the mean. $t$ test ${ }^{* * *} P<0.001$

The morphological changes of seminiferous tubules in rats with DM observed in the present study including separation of the germinal epithelium, vacuolization, luminal sloughing of germ cells, and atrophy of seminiferous tubules and may also result from hyperglycemia leading to high production of ROS. Oxidative stress has been reported to induce Leydig cell dysfunction and result in a testosterone deficiency, which plays an important role in spermatogenesis. Our present findings are consistent with those of previous studies, which found that DM induced the atrophy of seminiferous tubules by decreasing the number of spermatogenic cells $[7,8,26]$.

In the present study, a decrease in testicular diameter and testicular weight was observed in association with an increase in the atrophy of tubules in the rats in the DM group, which is consistent with findings reported previously [27, 28]. These decreases may be due to a decrease in testosterone levels leading to degeneration of the Sertoli-Sertoli cell junction, which is implicated in structural changes of seminiferous tubules $[29,30]$.

The significant decrease in the proportion of late stage (stage IX-XIV) seminiferous tubules in rats in the DM group may reflect an arrested period of development causing impairment of spermatogenesis. This decrease may be due to decreases in testosterone levels, dysfunction of Leydig cells, changes in structure and function of Sertoli cells, or atrophy of the seminiferous tubules $[17,29]$. The significant increase in the proportion of early stage tubules in rats in the DM group may also reflect nonprogressive development of the tubules due to an impairment of testosterone production from Leydig cells or changes in the structure and function of Sertoli cells.

The results of the present study are limited to type I DM, which involves impairment of insulin production. Altered fat metabolism with impaired insulin production occurs in type II DM. We cannot extrapolate the results to type II DM because we did not modify any parameters related to fat metabolism. The pathology of type II DM needs to be further elucidated. The numbers of rats in each group were not equal due to inclusion criteria for the rat model of diabetes. Nevertheless, the numbers were suitable for statistical analysis.

We reduced the number of rats used in biomedical research by using testis and epididymis from rats used for another study "Development of topical cream containing long peper extract 
loaded solid lipid microparticles on nerve pain relief in of rat model of diabetic neuropathy". Thus, by this dual-purpose subproject arrangement tissues and organs were collected for further studies such as of the histopathology reported here.

\section{Conclusions}

The present findings support previous evidence that DM has adverse effects on sperm quality, structural changes in testis, and seminiferous tubules, as well as changes in the development of seminiferous tubules during spermatogenesis. These changes may be caused by high production of ROS induced by hyperglycemia in the rat model of type 1 DM leading to impaired function of all structures involved in sperm production and development. Our findings may be beneficial for a greater awareness of the adverse effects of DM on reproductive function or for use as a model for the development of new therapeutic treatments for DM.

Author contributions. SN, NN, CM, SN-T, and ST contributed substantially to the conception and design of this study. NN and CM contributed substantially to the acquisition of data. All the authors analyzed and interpreted the data. SN, NN, and CM drafted the manuscript and SN-T and ST critically revised it. All the authors approved the final version submitted for publication and take responsibility for statements made in the published article.

Acknowledgments. This research was supported financially by the Thai Agricultural Research Development Agency (public organization) and Naresuan University Research Fund. The present work was presented in part as a poster at the 41st Annual Conference of the Anatomy Association of Thailand (AAT41), 2018 May 23-25, Cha-am, Phetchaburi, Thailand. Sureeporn Nak-ung, Nutnicha Nakprom, Chonikan Maneegam, Sutisa Nudmamud-Thanoi, Samur Thanoi. Changes of sperm quality and testicular structure in diabetic rats [PP 25, AAT158].

Conflict of interest statement. The authors have each completed and submitted an International Committee of Medical Journal Editors Uniform Disclosure Form for Potential Conflicts of Interest. None of the authors have any potential or actual conflict of interest to disclose.

\section{References}

[1] American Diabetes Association. 2. Classification and diagnosis of diabetes. Diabetes Care. 2015; 38 (Suppl 1):S8-16.
[2] Amaral S, Oliveira PJ, Ramalho-Santos J. Diabetes and the impairment of reproductive function: possible role of mitochondria and reactive oxygen species. Curr Diabetes. 2008; 4:46-54.

[3] Ullah A, Khan A, Khan I. Diabetes mellitus and oxidative stress-A concise review. Saudi Pharmaceutical Journal. 2016; 24:547-53.

[4] Baynes JW, Thorpe SR. Role of oxidative stress in diabetic complications: a new perspective on an old paradigm. Diabetes. 1999; 48:1-9.

[5] Agarwal A, Saleh RA, Bedaiwy MA. Role of reactive oxygen species in the pathophysiology of human reproduction. Fertil Steril. 2003; 79:829-43.

[6] Tremelln K. Oxidative stress and male infertility-a clinical perspective. Hum Reprod Update. 2008; 14:243-58.

[7] Ricci G, Catizone A, Esposito R, Pisanti AF, Vietri TM, Galdirei M. Diabetic rat testes: morphology and functional alterations. Andrologia. 2009; 41:361-8.

[8] Kanter M, Aktas C, Erboga M. Protective effects of quercetin against apoptosis and oxidative stress in streptozotocin-induced diabetic rat testis. Food Chem Toxicol. 2012; 50:719-25.

[9] Agarwal A, Viek G, Ong C, du Plessis SS. Effect of oxidative stress on male reproduction. World J Mens Health. 2014; 32:1-17.

[10] Baccetti B, la Marca A, Piomboni P, Capitani S, Bruni E, Petraglia F, De Leo V. Insulin-dependent diabetes in men is associated with hypothalamo-pituitary derangement and with impairment in semen quality. Hum Reprod. 2002; 10:2673-7.

[11] Kim ST, Moley KH. Paternal effect on embryo quality in diabetic mice is related to poor sperm quality and associated with decreased glucose transporter expression. Reproduction. 2008; 136:313-22.

[12] Navarro-Casado L, Juncos-Tobarra MA, Cháfer-Rudilla M, Íñiguez de Ozoño L, Blázquez-Cabrera JA, Miralles-García JM. Effect of experimental diabetes and STZ on male fertility capacity study in rats. J Androl. 2010; 31:584-92.

[13] Mangoli E, Talebi RA, Anvari M, Pourentezari M. Effect of experimentally-induced diabetes on sperm parameters and chromatin quality in mice. Iran J Reprod Med. 2013; 11:53-60.

[14] Lenzen S. The mechanisms of alloxan- and streptozotocin-induced diabetes. Diabetologia. 2008; 51:216-26.

[15] Mitani O, Masui K, Tsujimoto H, Jinbo K, Watanabe Y, Ohkura T. Histopathological changes of streptozotocin-induced painful diabetes and antihyperalgesic effect of capsaicin cream in rats. J Toxicol Pathol. 2008; 21:97-104.

[16] Mortimer D, Mortimer ST. Manual methods for sperm motility assessment. Methods Mol Biol. 2013; 927:61-75.

[17] Roboon J, Nudmamud-Thanoi S, Thanoi S. Recovery effect of pre-germinated brown rice on the alteration of sperm quality, testicular structure and androgen receptor expression in rat model of depression. Andrologia. 2017; 49:e12596; doi: 10.1111/and.12596.

[18] Wyrobek AJ, Bruce WR. Chemical induction of sperm abnormalities in mice. Proc Natl Acad Sci U S A. 1975; 72:4425-9.

[19] Nudmamud-Thanoi S, Thanoi S. Methamphetamine induces abnormal sperm morphology, low sperm concentration and apoptosis in the testis of male rats. Andrologia. 2011; 43:278-82.

[20] Nudmamud-Thanoi S, Thanoi S. Pseudoephedrine induces sperm abnormalities, lower sperm counts and increased apoptosis in rat testis. Cell Tissue Res. 2012; 349:625-30.

[21] Hess RA. Quantitative and qualitative characteristics of the stages and transitions in the cycle of the rat seminiferous epithelium: light microscopic observations of perfusion-fixed and plastic-embedded testes. Biol Reprod. 1990; 43:525-42. 
[22] Nudmamud-Thanoi S, Tangsrisakda N, Thanoi S. Changes of androgen receptor expression in stages VII-VIII seminiferous tubules of rat testis after exposure to methamphetamine. Songklanakarin J Sci Technol. 2016; 38:275-9.

[23] Amaral S, Moreno AJ, Santos MS, Seiça R, Ramalho-Santos J. Effects of hyperglycemia on sperm and testicular cells of Goto-Kakizaki and streptozotocin-treated rat models for diabetes. Theriogenology. 2006; 66: 2056-67.

[24] Aitken RJ, Clarkson JS, Fishel S. Generation of reactive oxygen species, lipid peroxidation, and human sperm function. Biol Reprod. 1989; 41:183-97.

[25] Kao SH, Chao HT, Wei YH. Multiple deletions of mitochondrial DNA are associated with the decline of motility and fertility of human spermatozoa. Mol Hum Reprod 1998; 4:657-66.
[26] Kanter M, Aktas C, Erboga M. Curcumin attenuates testicular damage, apoptotic germ cell death, and oxidative stress in streptozotocin-induced diabetic rats. Mol Nutr Food Res. 2013; 57:1578-85.

[27] Guneli E, Tugyan K, Ozturk H, Gumustekin M, Cilaker S, Uysal N. Effect of melatonin on testicular damage in streptozotocin-induced diabetes rats. Eur Surg Res. 2008; 40:354-60.

[28] Khaneshi F, Nasrolahi O, Azizi S, Nejati V. Sesame effects on testicular damage in streptozotocin-induced diabetes rat. Avicenna J Phytomed. 2013; 3:347-55.

[29] Foley G.L. Overview of male reproductive pathology. Toxicol Pathol. 2001; 29:49-63.

[30] Lui W-Y, Murk D, Lee WM, Cheng CY. Sertoli cell tight junction dynamics: their regulation during spermatogenesis. Biol Reprod. 2003; 68:1087-97. 
